\title{
NEUTRINO FACTORY BASED ON MUON-STORAGE-RINGS TO MUON COLLIDERS: PHYSICS AND FACILITIES
}

\begin{abstract}
Z. Parsa*, Brookhaven National Laboratory, Abstract

Intense muon sources for the purpose of providing intense high energy neutrino beams ( $\nu$ factory) represents very interesting possibilities. If successful, such efforts would significantly advance the state of muon technology and provides intermediate steps in technologies required for a future high energy muon collider complex. High intensity muon: production, capture, cooling, acceleration and multi-turn muon storage rings are some of the key technology issues that needs more studies and developments, and will briefly be discussed here. A muon collider requires basically the same number of muons as for the muon storage ring neutrino factory, but would require more cooling, and simultaneous capture of both $\pm \mu$. We present some physics possibilities, muon storage ring based neutrino facility concept, site specific examples including collaboration feasibility sutudies, and upgrades to a full collider.
\end{abstract}

\section{INTRODUCTION}

A neutrino factory based on a muon storage ring is a natural path to muon collider technology, since both facilities share essentially the same subcomponents prior to the storage ring. In the following, physics petentials, examples and feasibility studies for FNAL (study-1) and BNL (study-2) site specific muon storage rings are given.

\section{PHYSICS POTENTIALS}

The increasing interest in the Neutrino oscillation physics span from the solar neutrino deficit and the evidence for $\nu_{\mu} \rightarrow \nu_{e}$ oscillations (from the LSND experiment), to the exciting atmospheric neutrino results including measurements of the atmospheric Muon - Neutrino deficit from the SuperK (Superkamiokande) experiment that has provided convincing evidence for lepton number violation. The next generation of long baseline experiments such as $\mathrm{K} 2 \mathrm{~K}$, MINOS, OPERA, ICANOE are expected to confirm e.g., the $\nu_{\mu} \rightarrow \nu_{e}$ oscillations fully or partially, etc. And LSND, SNO and KamLAND, together with further SuperK measurements are expected to (rule out or) provide us with one viable region of parameter space for solar neutrino oscillations. But there still will remain many unanswered basic questions. To answer these unanswered questions will require a new generation of beams and detectors beyond the next generation of experiments. Fig. 1, shows the current and expected limits for some of the future neutrino oscillation experiments, (here different oscillation modes are shown together)[2]. A Nu-

\footnotetext{
* Supported by US Department of Energy contract Number DE-AC0298CH10886. E-mail: parsa@bnl.gov
}

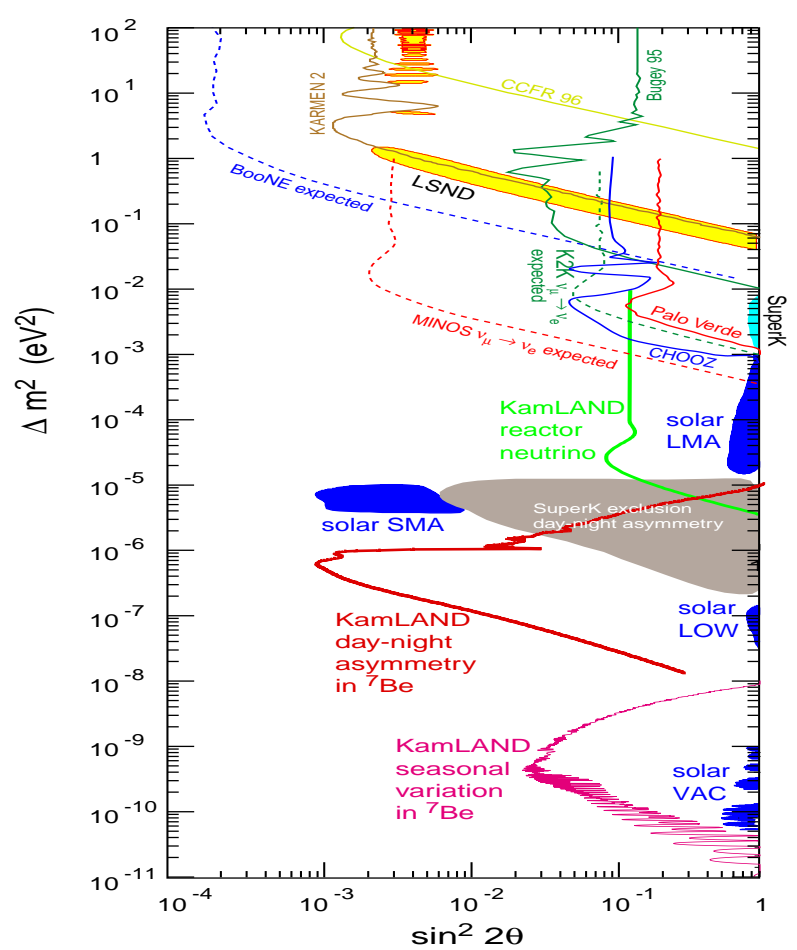

Figure 1: Shows some expected and current limits for future neutrino oscillation experiments.

trino Factory based on Muon storage ring(s) can be a first phase toward building a high energy Muon Collider. The Physics program of a Neutrino Factory would focus on the relatively unexplored neutrino sector and would make valuable contributions to oscillation studies. Fig.2, shows plot of the Muon Decays per year $\left(10^{7}\right.$ seconds $)$ vs., Muon Energy in $\mathrm{GeV}$. Assuming a $50 \mathrm{kT}$ detector, it includes the required flluxes for various physics searches [2]. This figure shows ranges of physics possibilities with neutrino beams from various muon storage facility designs, e.g., for the study-1 (50 GeV muons and 1.5 MW) and collaboration study-2 (20 GeV muons at $1 \mathrm{MW}$ and Upgrade to $4 \mathrm{MW}$ ). The neutrino flux produced, e.g. in a $20 \mathrm{GeV}$ Muon storage ring, is expected to have an average energy of about $14 \mathrm{GeV}$ at the detector (?).

\section{NEUTRINO FACTORY HAS LESS STRINGENT REQUIREMENTS COMPARED TO A MUON COLLIDER}

A neutrino factory based on a muon storage ring is a challenging extension of present accelerator technology. Conventionally, neutrino beams employ a proton beam on a target to generate pions, which are focused and allowed 


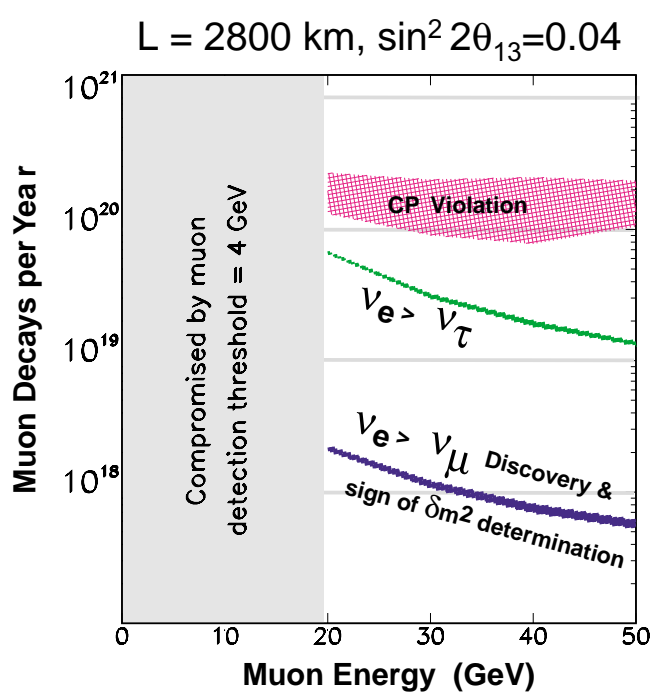

Figure 2: Muon Decays per year $\left(10^{7}\right.$ seconds $)$ vs., Muon Energy showing various physics searches.

to decay into neutrinos and, muons The muons are stopped in the shielding, while the muon-neutrinos are directed toward the detector. In a neutrino factory, pions are made the same way and allowed to decay, but it is the decay muons that are captured and used. The initial neutrinos from pion decay are discarded, or used in a parasitic lowenergy neutrino experiment. But the muons are accelerated and allowed to decay in a storage ring with long straight sections. It is the neutrinos from the decaying muons (both muon-neutrinos and anti-electron-neutrinos) in the straight sections, that are directed to a detector. The Storage ring effectiveness $\left(S_{e f f}\right)$ is ratio of the lengths of the straight section $\left(L_{s}\right)$ to the ring circumference $(C)$. For example, for the ring geometry in Fig. 3, (with the arc length $L_{\text {arc }}$ ), the storage ring effectiveness is: $S_{\text {eff }}=L_{s} / 2\left(L_{s}+L_{\text {arc }}\right)$.

In a neutrino factory, a proton driver of moderate energy ( $<50 \mathrm{GeV})$ and high average power,(e.g., 1-4 MW), similar to that required for a muon collider, but with a less stringent requirements on the charge per bunch and power is needed. This is followed by a target and a pion-muons capture system. A longitudinal phase rotation is performed to reduce the muon energy spread at the expense of spreading it out over a longer time interval.

The phase rotation system may be designed to correlate the muon polarization with time, allowing control of the relative intensity of muon and anti-electron neutrinos. Cooling is used to reduce phase space, e.g., about a factor of 50 in six dimensions. This is much smaller than the factor of $10^{6}$ needed for a muon collider. Production is followed by fast muon acceleration to $50 \mathrm{GeV}$ for example of Feasibility study-1, and $20 \mathrm{GeV}$ for Brookhaven - site feasibility study-2, in a system of linac and (e.g., two) recirculating linear accelerators (RLA's), which may be identical to that for a first stage of muon collider such as a Higgs Factory[1]. A muon-storage ring with long straight sections could point to one or more distant neutrino detectors for oscillation studies, and to one or more near detectors for high intensity scattering studies. Fig. 3 illustrates a simple racetrack-shaped configuration, with two long straight sections.

\section{FEASIBILITY OF MUON STORAGE RING FACILITIES?}

Attempts [4],[1]-[5] were made to investigate the physics and technical feasibility of muon storage rings. First for a $50 \mathrm{GeV}$ energy, FNAL-site specific ring, followed by studies for a $20 \mathrm{GeV}$ BNL - based ring. Fig. 4, shows footprint of the whole facility for FNAL[4] (that also can fit on other sites). This shows various subsystems to scale on a site of abouut $2 \mathrm{Km}$ by $1 \mathrm{~km}$. With a $50 \mathrm{GeV}$ beam energy and average beam power of $\sim 240 \mathrm{KW}$. The storage ring acceptance was designed for $3 \sigma$ of $3.2 \pi^{*} \mathrm{~m}^{*} \mathrm{rad}$, to allow for emittance growth of about a factor of two in the accelerating systems once the muon beam has been cooled down to the goal value of $1.6 \pi^{*} m^{*} \mathrm{rad}$. Table 1 shows the final Parameters for the FNAL-site Muon Ring studies. For (FNAL-site) study-1, a $16 \mathrm{GeV}$ proton (driver) beam was used while in the (BNL-site) study-2, the AGS $24 \mathrm{GeV}$ proton beam and its upgrade are considered.

Table 1: Final Parameters for the $50 \mathrm{GeV}$ Muon Storage.

\begin{tabular}{|l|c|}
\hline Energy [GeV] & $\mathbf{5 0}$ \\
\hline Decay ratio per straight [\%] & 39 \\
Designed inv. Emittance $\left[\pi^{*} \mathrm{~m}^{*}\right.$ rad] & 0.0032 \\
Emittance at Cooling exit $\left[\pi^{*} \mathrm{~m}^{*}\right.$ rad] & 0.0016 \\
$\beta_{0}$ in straight $[\mathrm{m}]$ & 440 \\
$N_{\mu} /$ pulse $\left[10^{12}\right]$ & 2 \\
Decay angle of $\mu=1 / \gamma[\mathrm{mrad}]$ & 2.0 \\
Beam angle $\left(\sqrt{\epsilon} / \beta=\sqrt{\epsilon} \gamma_{\perp}\right) \quad[\mathrm{mrad}]$ & 0.2 \\
Lifetime $c^{*} \gamma^{*} \tau \quad[\mathrm{m}]$ & $3 \times 10^{5}$ \\
$\frac{\gamma_{\perp}=\left(1-\alpha^{2}\right)}{\beta_{0}}$ & \\
\hline
\end{tabular}

Following the $50 \mathrm{GeV}$ study-1, illustrated in

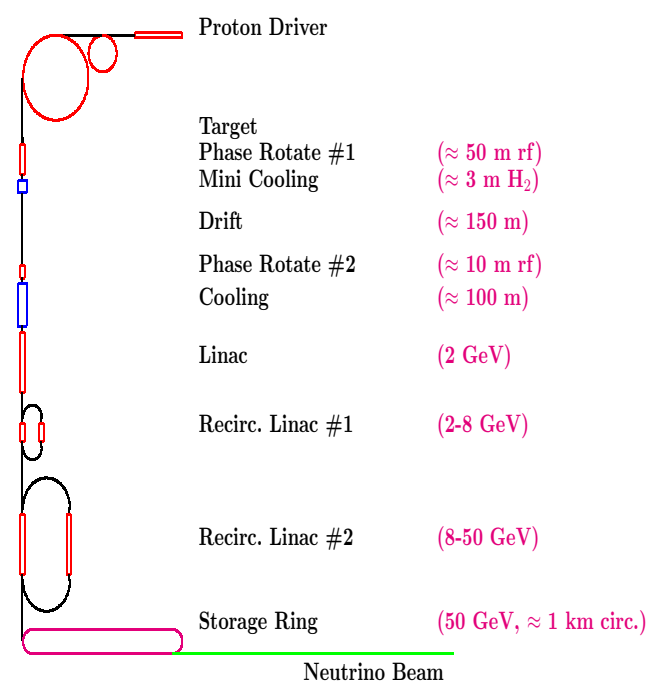

Figure 3: Overview of a Neutrino Factory Concepts . 


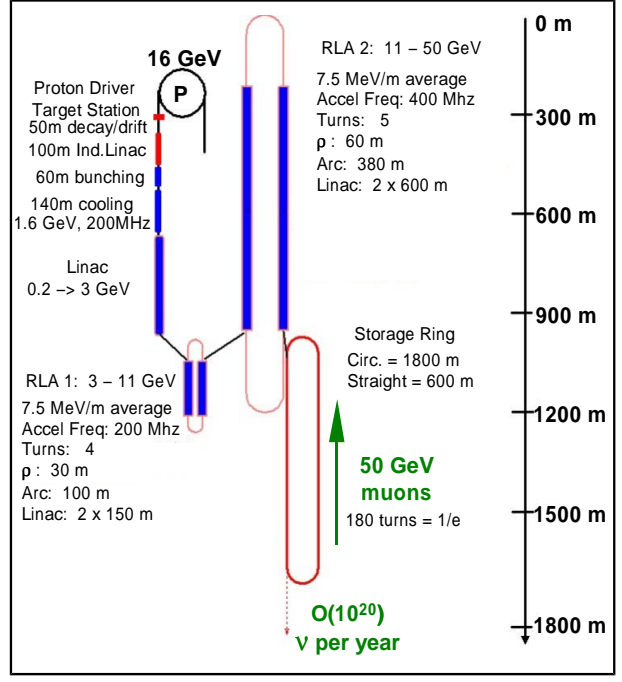

Figure 4: Footprint of a $50 \mathrm{GeV}$ Facility.

Fig. reffig:NUF-Scheme16GeV.eps [4], our collaboration aimed at a lower cost (thus lower energy) $20 \mathrm{GeV}$ muon storage ring (for 1 and $4 \mathrm{MW}$ proton powers) at BNL [4]. Mimicing the schematic shown in Fig. 3 the layout for the study-1 includes the following subsystem (sequence): A (200 MeV) Warm Linac; followed by a $(1 \mathrm{GeV})$ Superconducting Linac; a (3ns rms) Bunch Compressor; a $(0.45 \mathrm{~m}) \mathrm{Hg}$ Target; $(100 \mathrm{~m})$ Induction Linac\# 1; a ( $3.5 \mathrm{~m} \mathrm{H}_{2}$ ) mini Cooling section; an $(80 \mathrm{~m})$ Induction Linac\# 2; another $80 \mathrm{~m}$ Induction Linac\# 3; a (55 m) Buncher, then (108 m) cooling, a $2.3 \mathrm{GeV}$ Linac, a (2.3-20GeV) Ricirculating Accelerator Linac (RLA) and a $20 \mathrm{GeV}$ Storage Ring. Note, these parametrs will change, depending on simulation codes, followup studies and system optimization.

The proton driver for BNL (study-2), is an upgrade of the Brookhaven Alternating Gradient Synchrotron (AGS). Where a $1.3 \mathrm{GeV}$ superconducting proton linac replaces the current AGS - booster and the repetition rate is increased from $0.5 \mathrm{~Hz}$ to $2.5 \mathrm{~Hz}$. The expteced average power is $1 \mathrm{MW}$, and an upgrade to $4 \mathrm{MW}$ would require about $2 \times 10^{14}$ ppp and $5 \mathrm{~Hz}$, and may also require a superconducting bunch compressor, etc.[4]. The BNL-design uses Mercury (instead of Carbon) target, and 3 phase rotation induction linacs. For Acceleration a $(2.82 \mathrm{GeV}, 433 \mathrm{~m})$ linac followed by a single recirculator linac (that raises the energy to $20 \mathrm{GeV}$ in 4 passes) is used. After acceleration, the muons get injected into a racetrack shaped storage ring (with $358.18 \mathrm{~m}$ circumference). To minimize arc lengths and increase the number of muons decaying into neutrinos (in the downward - straight section, of length $=126 \mathrm{~m}$ ), in the detector direction, high field superconductor magnets are used in the arcs. From simulation, final number of muons delivered to the storage ring is about $3.5 \times 10^{20}$ for the $1 \mathrm{MW}$ proton driver; and about $1.4 \times 10^{21}$ for the $4 \mathrm{MW}$ proton driver, [4]. Which indicates some enhancement over the earlier Study-1 results.

Some of the detector - sites, for this study could include: MINOS detector in Sudan located at about 1,713 Km from BNL; Homestake mine in South Dakota at about 2,528 Km from BNL; and the WIPP facility in Carlsbad in New Mexico at about 2,903 Km from BNL. Noting that since at BNL-site, the water-table averages about 48 feet ( \pm 5 variation), and the highest ground elevation (for placing the muon ring) is about 90 feet above the sea level, with the bottom of the storage ring at 63 feet above the sea level, a hill would be needed (to be built?) to cover the entire storage ring (underground). E.g., for the straight section (for this neutrino beam) to point in the direction of a detector at WIPP (facility in Carlsbad), the storage ring must be tilted by about 13.1 degrees with respect to the horizantal direction[4].

\section{DISCUSSIONS}

The BNL-site specific $20 \mathrm{GeV}$ muon storage ring facility (study-2) shows an enhanced performance over the earlier Study-1, which it was based on. But there still remaines (both technical and fiscal) challenges. E.g., in the BNL-site design the proton driver would be an upgrade of the AGS, that although it would not technically be a major problem, it may be costly. Other more difficult technical components include: mercury jet target; a high radiation environment which requires remote handling; cooling (would be helpful), etc. progress continues to be made. Other intermediate steps such as, high intensity muon experiments (as BNL- MECO) shuld be supperted as they will greatly expand our abilities and confidence in credibility of higher energy colliders. due to space limitations, additional information on Cooling and upgrades to e.g. Higgs Factory Muon Collider(s) is given in the followup presentations[1].

\section{REFERENCES}

[1] Z. Parsa "Neutrino Factory - Ionization Cooling and Emittance Exchange"; ibid "Higgs Factory and Potentials" presentaions at PAC2001 (to be published).

[2] S. Geer, Phys. Rev. D 57, 6989 (1998); Collaboration page: www.cap.bnl.gov/mumu; K.T. McDonald, ed. physics/9911009, and references.; C. Albright, et al., Physics at a Neutrino Factory FERMILAB-FN-692, (2001).

[3] Z. Parsa, Muon Storage Rings - Neutrino Factories, in Next Generation Nucleon decay and Neutrino detector (99), ed. M. Diwan and C. Jung, AIP CP 533, pp. 181-195, N.Y. (2000) References therein.

[4] N. Holtkamp, Proc. EPAC2000, Vienna, Austria (2000); Neutrino Factory Feasibility Study-1: http://www.fnal.gov/projects/muon_collider/nu_factory/; Neutrino Factory Feasibility Study-2: http://www.cap.bnl.gov/mumu/studyii/

[5] Z. Parsa and W. J. Marciano (Editors), Proc. Intersections of Particle and Nuclear Physics 7th Conference, Qubec City, Canada 22-28 May 2000. AIP CP 5491019 pages, American Institute of Physics, NY (2000). 